\title{
CALIDAD DE VIDA VINCULADA A SALUD EN POBLACIÓN MIGRANTE RURAL-URBANA Y POBLACIÓN URBANA EN LIMA, PERÚ*
}

\author{
Gianmarco Márquez-Montero',a, Christian Loret de Mola ${ }^{2,3, b}$, Antonio Bernabé-Ortiz ${ }^{1,2, c}$, \\ Liam Smeeth ${ }^{2,4, d}$, Robert H. Gilman 2,5,6,e J. Jaime Miranda2,4,7,d
}

\begin{abstract}
RESUMEN
Objetivos. Explorar si existe una diferencia en la percepción y autorreporte de calidad de vida en población migrante rural-urbana en comparación con un grupo urbano no migrante. Materiales y métodos. Estudio transversal, análisis secundario del estudio PERU-MIGRANT (PEru's Rural to Urban MIGRANTs study). Utilizando la prueba de KruskallWallis y evaluando magnitud de efectos se midieron y compararon los puntajes global y por dominios obtenidos en la encuesta WHOQOL-Brief. Resultados. Se encuestó a 307 individuos. La edad promedio de fue de 47 años, $57 \%$ correspondieron al sexo femenino y $62,2 \%$ a población migrante. Comparado con el grupo urbano, el grupo migrante reportó puntajes menores de calidad de vida a nivel global y en los dominios de salud psicológica y del ambiente en el que viven y a la vez, reportaron una mejor percepción en el dominio de salud física. Conclusiones. El impacto de la migración rural-urbana en la calidad de vida indica un efecto diferencial según sus distintos dominios.
\end{abstract}

Palabras clave: Migración Interna; Migración Rural-Urbana; Calidad de Vida; Perú; Países en Desarrollo (fuente: DeCS BIREME).

\section{HEALTH-RELATED QUALITY OF LIFE AMONG URBAN AND RURAL TO URBAN MIGRANT POPULATIONS IN LIMA, PERU}

\begin{abstract}
Objectives. To explore if there is a difference in the perception and self reported quality of life between rural-to-urban migrants and urban groups. Materials and methods. Cross-sectional study, secondary analysis of the PERU-MIGRANT study (PEru's Rural to Urban MIGRANTs Study). WHOQOL-Brief survey's global scores and per specific domains obtained in the survey were compared using Kruskall-Wallis' test and assessing size effect. Results. A total of 307 subjects (62.2\% migrants, 57\% female, means age 47 years-old) were surveyed. Compared with the urban group, migrants reported lower quality of life both on the global scores as well as in psychological health and the living environment domains. Migrants reported a higher score on the physical health's domain. Conclusions. The impact of rural-to-urban migration on quality of life suggests a differential effect within its specific domains.
\end{abstract}

Key words: Internal Migration; Rural-Urban Migration; Quality of Life; Peru; Developing Countries, (source: MeSH NLM).

\section{INTRODUCCIÓN}

A inicios de la década de los años 90, la Organización Mundial de la Salud (OMS) define la calidad de vida como: La percepción del individuo sobre su posición en la vida dentro del contexto cultural y el sistema de valores en el que vive $y$ con respecto a sus metas, expectativas, normas y preocupaciones (1). En las últimas décadas el concepto de calidad de vida ha venido tomando un importante impulso y se constituye como una metodología comúnmente utilizada para evaluaciones de calidad. Adicionalmente, este concepto ha demostrado tener capacidad predictiva tanto para la utilización de los servicios de salud como para morbi-mortalidad en diferentes enfermedades ${ }^{(2-4)}$. En tal sentido, la calidad de vida se constituye como un indicador, reconocido internacionalmente, de resultado o de necesidad en salud.

Las poblaciones migrantes con una exposición alta a violencia política, presentan una salud mental y calidad de vida menor ${ }^{(5)}$. Este antecedente genera una pregunta

\footnotetext{
Facultad de Salud Pública y Administración "Carlos Vidal Layseca”, Universidad Peruana Cayetano Heredia, Lima, Perú.

CRONICAS, Centro de Excelencia en Enfermedades Crónicas, Universidad Peruana Cayetano Heredia, Lima, Perú.

Mental Health Working Group, Universidad Peruana Cayetano Heredia, Lima, Perú.

4 Faculty of Epidemiology and Population Health, London School of Hygiene and Tropical Medicine. London, United Kingdom.

Department of International Health, Johns Hopkins Bloomberg School of Public Health. Baltimore, MD, USA.

6 Área de Investigación y Desarrollo, A. B. PRISMA, Lima, Perú.

7 Facultad de Medicina "Alberto Hurtado", Universidad Peruana Cayetano Heredia, Lima, Perú.

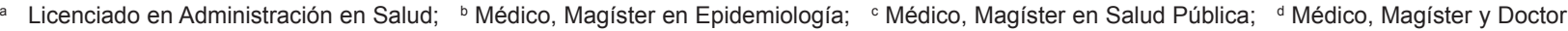
en Epidemiología; e Médico, Especialista en Enfermedades Infecciosas.

* Este trabajo se basa en la Tesis de Bachiller de Gianmarco Márquez Montero para optar el título de Licenciado en Administración en Salud en la Facultad de Salud Pública y Administración "Carlos Vidal Layseca”, Universidad Peruana Cayetano Heredia.
} 
relevante para nuestro medio, el cual ha sufrido procesos importantes de migración interna, y que podrían impactar en la calidad de vida de pobladores migrantes.

En el Perú, el periodo de violencia política de los años 80 se focalizó inicialmente en el departamento de Ayacucho. Se estima que un millón de personas (aproximadamente 200000 familias) fueron desplazadas por la violencia política, $\left(23 \%\right.$ de procedencia ayacuchana) ${ }^{(6)}$. Esta migración masiva estuvo vinculada con la necesidad de supervivencia y no se dio únicamente por razones económicas. Muchosmigrantesdesplazadosseinstalaron en los llamados "pueblos jóvenes" o "asentamientos humanos" en las periferias de Lima Metropolitana (7). El estudio del PERU MIGRANT fue diseñado teniendo en cuenta la violencia política como un factor propulsor de migración ${ }^{(8)}$ y el contexto sociopolítico peruano.

El presente estudio tiene por objetivo explorar si existe una diferencia en la percepción y auto reporte de calidad de vida de los individuos migrantes y no migrantes. Dada la magnitud de los procesos migratorios observados en nuestro medio, se justifica el tener alcances a dicho objetivo en el contexto peruano.

\section{MATERIALES Y MÉTODOS}

\section{DISEÑO}

Estudio transversal, análisis secundario del estudio PERU-MIGRANT, cuya metodología ha sido previamente detallada ${ }^{\left({ }^{(8)}\right.}$. Para este estudio se consideraron dos de las poblaciones originales del estudio PERU MIGRANT.

\section{POBLACIÓN DE ESTUDIO}

El estudio PERU MIGRANT se llevó a cabo en el distrito de San Juan de Miraflores en el año 2007. Los participantes nativos urbanos o limeños fueron seleccionados aleatoriamente de un censo local llevado a cabo en el año 2000.

La población migrante - definida como aquellas personas nacidas en Ayacucho, que habían migrado hacia Lima y que reportaban residir permanentemente en la comunidad de Pampas de San Juan de Miraflores- fue seleccionada aleatoriamente. Esta selección fue hecha tomando como marco muestral una actualización, llevada a cabo en el año 2006, del censo original (año 2000) con el fin de tener una identificación más exacta de este grupo de interés.

Los participantes del estudio fueron sujetos de ambos sexos, mayores de 30 años, excluyéndose a las mujeres embarazadas. El presente subestudio de calidad de vida $(n=307)$ se llevó a cabo en una submuestra de pobladores migrantes $(n=191)$ y no migrantes $(n=116)$ del estudio PERU MIGRANT. Los marcos muestrales del estudio original para el grupo de migrantes y urbanos fueron 600 y 200 sujetos, respectivamente. Para este subestudio, fueron invitados a participar los primeros 307 individuos seleccionados para el estudio global.

\section{VARIABLES E INSTRUMENTOS}

La variable principal estudiada fue calidad de vida, autorreportada por el participante, evaluada usando el cuestionario WHOQOL-Brief de 26 ítems ${ }^{(9,10)}$. Este cuestionario captura la percepción del participante en condiciones subjetivas y objetivas de su vida. Esta herramienta, desarrollada por la OMS, fue diseñada para crear un instrumento que pudiese comparar la calidad de vida en diferentes poblaciones. EI WHOQOL-Brief en su escala global tiene una puntuación del 1 al 5 , una mayor puntuación global refleja una mejor calidad de vida. Aparte, este instrumento mide los dominios de salud física, salud mental, relaciones sociales y ambiente, los cuales pueden ser analizados de manera independiente. Los puntajes según dominios de la herramienta utilizada en este estudio fueron transformados a su equivalente en la herramienta de 100 preguntas (WHOQOL-100) siguiendo procedimientos establecidos por la OMS ${ }^{(11)}$, y permitiendo hacer comparaciones con otros estudios que hayan utilizado la herramienta original.

Las variables sociodemográficas consideradas fueron edad, sexo, número de años de estudio completados (variable continua), nivel de educación, acceso a servicios de agua (presencia o ausencia de servicio de agua potable dentro de la vivienda como fuente primaria de acceso al agua), desagüe (presencia o ausencia de acceso a una red de desagüe dentro de la vivienda), combustible utilizado para cocinar (uso o no uso de gas), condiciones de la vivienda (techo, piso y paredes de material noble o no) y estado laboral (tener en la actualidad un empleo o no).

\section{ASPECTOS ÉTICOS}

El protocolo del estudio fue revisado y aprobado por el Comité de Ética de la Universidad Peruana Cayetano Heredia. Todos los participantes brindaron su consentimiento para participar.

\section{ANÁLISIS DE DATOS}

Se llevó a cabo un análisis descriptivo de los puntajes de calidad de vida obtenido según grupo de interés migrante o no migrante. Se utilizó la prueba no paramétrica de Kruskal-Wallis para las comparaciones de las puntuaciones obtenidas para calidad de vida, tanto global como por dominios, en cada grupo. 
Tabla 1. Características sociodemográficas de los grupos migrante y urbano.

\begin{tabular}{|c|c|c|c|}
\hline Variables & $\begin{array}{l}\text { Urbano } \\
(n=116)\end{array}$ & $\begin{array}{c}\text { Migrante } \\
(n=191)\end{array}$ & $\begin{array}{c}\text { Total } \\
(\mathrm{n}=\mathbf{3 0 7})\end{array}$ \\
\hline Edad $( \pm D E)$ & $44,1( \pm 9,5)$ & $49,3( \pm 12,3)$ & $47( \pm 0,7)$ \\
\hline Mujeres (\%) & $68(59 \%)$ & $107(56 \%)$ & $175(57 \%)$ \\
\hline Trabajan actualmente (\%) & $88(76 \%)$ & $149(78 \%)$ & $235(77 \%)$ \\
\hline Años de Estudio ( $\pm \mathrm{DE}$ ) [rango] & $10,1( \pm 3,5)[0-18]$ & $7,3( \pm 4,5)[0-17]$ & $8,3( \pm 4,4)[0-18]$ \\
\hline \multicolumn{4}{|l|}{ Nivel educativo (\%) } \\
\hline Sin educación formal & $1(1 \%)$ & $20(10 \%)$ & $17(6 \%)$ \\
\hline Primaria incompleta & $7(6 \%)$ & $41(22 \%)$ & $48(16 \%)$ \\
\hline Primaria completa & $12(10 \%)$ & $26(14 \%)$ & $38(12 \%)$ \\
\hline Secundaria incompleta & $32(28 \%)$ & $39(20 \%)$ & $71(23 \%)$ \\
\hline Secundaria completa & $30(26 \%)$ & $39(20 \%)$ & $69(23 \%)$ \\
\hline Superior incompleta & $17(15 \%)$ & $12(6 \%)$ & $29(10 \%)$ \\
\hline Superior completa & $17(15 \%)$ & $14(7 \%)$ & $31(10 \%)$ \\
\hline \multicolumn{4}{|l|}{ Tipo de abastecimiento } \\
\hline Agua en la vivienda & $108(93 \%)$ & $179(94 \%)$ & $287(94 \%)$ \\
\hline Desagüe en la vivienda & $108(93 \%)$ & $178(93 \%)$ & $286(93 \%)$ \\
\hline Uso de gas para cocción & $108(93 \%)$ & $168(88 \%)$ & $276(90 \%)$ \\
\hline \multicolumn{4}{|l|}{ Condiciones de la Vivienda } \\
\hline Techo de material concreto & $74(64 \%)$ & $118(62 \%)$ & $192(63 \%)$ \\
\hline Pared de ladrillos & $101(87 \%)$ & $164(86 \%)$ & $265(86 \%)$ \\
\hline Piso de cemento no acabado & $63(54 \%)$ & $104(55 \%)$ & $167(54 \%)$ \\
\hline
\end{tabular}

En el ámbito de calidad de vida es importante aproximarse a establecer si las diferencias observadas son clínicamente relevantes, siendo la magnitud de los efectos (effect sizes en Inglés) uno de los estimados utilizados ${ }^{(12-15)}$. La magnitud de los efectos para las diferencias en los puntajes globales y por dominios entre los dos grupos de interés fue calculado dividiendo las diferencias de los valores promedios entre la desviación estándar (DE) del grupo de comparación (13). Este estimado permite que los resultados sean comparables entre distintos dominios. Se consideró la aproximación (rule of thumb) de Cohen para la interpretación de estos efectos: 0,20 representa un efecto pequeño, 0,50 un efecto moderado $y \geq 0,80$ un efecto mayor ${ }^{(16)}$.

\section{RESULTADOS}

\section{DATOS SOCIODEMOGRÁFICOS}

En total participaron 307 personas, 191 (62,2\%) migrantes y $116(37,8 \%)$ del grupo urbano. La edad promedio fue de 47 años (rango 30-88), 57\% fueron de sexo femenino y $77 \%$ reportó tener un trabajo en la actualidad. El grupo migrante tuvo, en promedio, 3 años de estudio menos que el grupo urbano. Los niveles educativos alcanzados fueron diferentes en los grupos estudiados: por ejemplo, $10 \%$ frente al $1 \%$ de ausencia de educación formal en población migrante y urbana, respectivamente. Las características sociodemográficas de los participantes se presentan en la Tabla 1. No se observó marcadas diferencias con respecto a las condiciones de viviendas y acceso a servicios.

\section{ANÁLISIS BIVARIADO}

El resultado de los análisis bivariados entre los diferentes dominios de la WHOQOL-BRIEF se presenta en la Tabla 2. Hubo una fuerte evidencia de diferencias en puntajes de calidad de vida promedio entre grupos migrantes y urbano. La autopercepción de calidad de vida a nivel global, en el dominio psicológico y en el dominio de ambiente fue mayor en el grupo no migrante urbano. Por el contrario, la puntuación en el dominio físico fue mayor en el grupo migrante. No se observó diferencias en el dominio de relaciones sociales en los grupos estudiados. La magnitud de los efectos para el puntaje

Tabla 2. Distribución de resultados de puntuación en el cuestionario WHOQOL-Brief.

\begin{tabular}{|c|c|c|c|c|}
\hline & $\begin{array}{c}\text { Urbano } \\
\text { Media ( } \pm D E)\end{array}$ & $\begin{array}{c}\text { Migrante } \\
\text { Media (士DE) }\end{array}$ & $p^{*}$ & Magnitud del efecto (DE) \\
\hline Calidad de Vida Global & $3,18( \pm 0,45)$ & $3,00( \pm 0,39)$ & $<0,001$ & $-0,40$ \\
\hline Dominio Físico & $69,55( \pm 13,16)$ & $73,28( \pm 11,76)$ & 0,012 & $+0,28$ \\
\hline Dominio Psicológico & $65,94( \pm 12,76)$ & $63,05( \pm 12,42)$ & 0,026 & $-0,23$ \\
\hline Dominio Relaciones Sociales & $65,49( \pm 13,18)$ & $65,46( \pm 14,05)$ & 0,701 & $-0,002$ \\
\hline Dominio Ambiente & $52,19( \pm 11,42)$ & $48,28( \pm 10,76)$ & 0,001 & $-0,34$ \\
\hline
\end{tabular}

Nota: Calidad de vida global: puntuación de 1 a 5; Dominios: puntuación de 0 a 100.

*valor de $\mathrm{p}$ obtenido mediante prueba no paramétrica de Kruskal-Wallis. 
global de calidad de vida y para el dominio de medio ambiente fue $-0,4$ y $-0,34 \mathrm{DE}$, respectivamente.

\section{DISCUSIÓN}

El objetivo del presente estudio fue explorar la calidad de vida en grupos migrantes y urbanos no migrantes. En tal sentido se observó diferencias significativas entre la percepción global de calidad de vida entre migrantes y urbanos, así como diferencias en los componentes de salud física, psicológicos y de ambiente, mas no sobre las relaciones sociales. Sin embargo, sumando complejidad a las observaciones, las diferencias entre los grupos no fueron uniformes. Es decir, mientras algunos componentes tuvieron puntuación mayor en el grupo urbano, ocurrió lo opuesto con el dominio de salud física.

Al ser los migrantes procedentes de áreas con niveles más altos de actividad física ${ }^{(17)}$, es posible que estén más acostumbrados o familiarizados con actividades que requieren esfuerzo físico. En tal sentido, es posible postular que la población migrante tenga una percepción distinta frente a esfuerzos habituales comparada con el grupo urbano. A pesar de que la población migrante puntúa mejor en el dominio físico, lo mismo no ocurre con el dominio psicológico. Esta discrepancia podría estar vinculada con una serie de factores externos o emocionales, que involucran una variedad de aspectos vinculados con la decisión de migrar en un contexto de violencia política y el impacto de la migración en iniciar la adaptación a un contexto distinto en el ámbito urbano.

Estas observaciones diferenciales en distintos dominios de calidad de vida indican que el impacto de la migración ruralurbana es más complejo de lo que se asumiría previamente. La diferencia en la magnitud de los efectos encontrados, que van en ambas direcciones, sugiere que las diferencias observadas son pequeñas, pero que, comparado con otras condiciones, podrían considerarse como clínicamente relevantes ${ }^{(5,13)}$. De hecho, la magnitud de los efectos en la calidad de vida observados en este estudio postmigración rural-urbana son similares a los descritos en población Latinoamericana con exposición previa a violencia política que migraron a Estados Unidos de Norteamérica ${ }^{(5)}$.

Los hallazgos del presente estudio requieren enmarcarse en los continuos procesos migratorios que han ocurrido y continúan ocurriendo en nuestra región. Estos procesos migratorios involucran tanto a la migración interna así como a la migración externa o internacional.

La urbanización en los últimos 150 años han permitido importantes cambios en la educación, nivel de empleo, acceso a servicios de salud, vivienda y más ${ }^{(18)}$. En el año 2002, el número de personas que residían fuera de su país de origen fue de 175 millones; esta cantidad es más del doble de aquella descrita en los años $70{ }^{(19)}$. Se estima que casi 20 millones de personas naturales de la región de América Latina y el Caribe residen fuera de su país de origen. Esta cifra equivale alrededor del $13 \%$ del total de migraciones internacionales en todo el mundo (19). La mitad de estas migraciones se realizaron durante el decenio del 90, en particular a los Estados Unidos y, posteriormente, a Europa ${ }^{(20)}$. Los procesos migratorios imponen retos importantes ${ }^{(21-23)}$ y sus efectos en la salud de los migrantes no son muy claros ${ }^{(24)}$.

Asimismo, América Latina sigue un proceso de desarrollo importante en lo que a urbanización concierne. Actualmente, más del $60 \%$ de la población de la región habita en centros urbanos ${ }^{(25)}$ y se estima que para el 2020 un $80 \%$ del total de la población Latinoamericana vivirá en centros urbanos ${ }^{(26)}$. Parte de este crecimiento poblacional en zonas urbanas es atribuible a migraciones internas ${ }^{(27)}$, tal y como ocurrió en el caso del Perú en la década de los $80^{(7,28)}$. En tal sentido, llama la atención que los migrantes perciban un mejor nivel de salud física, mientras que ocurre lo contrario con los otros dominios a pesar de que ambas poblaciones viven en el mismo espacio geográfico, en la mayoría de casos por más de 20 años. A pesar de que el tamaño de muestra del presente estudio es pequeño, este trabajo aborda un tema relevante y poco divulgado que justifica su relevancia para nuestro y otros contextos.

No existen muchos reportes de calidad de vida en procesos migratorios internos, menos aun, migración rural-urbana, haciendo más relevante los resultados de este análisis. Solo encontramos un estudio que evaluó la calidad de vida luego de migración ruralurbana en China, siendo el grupo de comparación los no-migrantes rurales, lo que limita la comparación con nuestros resultados. Este estudio encontró que los migrantes reportaron una menor calidad de vida y mayor estigmatización que sus contrapartes rurales ${ }^{(29)}$.

En relación a calidad de vida y migración internacional, reportes de otros estudios ${ }^{(5,30,31)}$ sugieren que las personas migrantes tienden a tener menor puntaje en los índices de percepción de calidad de vida o salud autorreportada. Sin embargo esta observación no se mantiene en todas las investigaciones y otros autores plantean el fenómeno del "migrante saludable" indicando que en el primer momento de llegada y hasta pasados los diez primeros años de estancia, la salud y la auto percepción de la población inmigrante suelen ser mejores ${ }^{(32-35)}$.

Vale la pena notar que en el proceso de migración internacional participan diversos factores que limitan la com- 
paración directa de nuestros resultados. Estos reportes se enfocan en diferentes temas dentro de la migración, como el lenguaje ${ }^{(30)}$, violencia ${ }^{(5)}$ y aculturación ${ }^{(31)}$. Algunos de estos estudios reportados utilizaron la Short Form Health Survey (SF-36) ${ }^{(5,30,36)}$, que a pesar de ser una herramienta diferente, tiene dominios similares al WHOQOL-Brief.

Asimismo, el análisis realizado, de índole exploratoria, no toma en cuenta posibles variables de confusión que podrían estar afectando las diferencias encontradas. Es posible que otras variables exploradas en procesos de migración internacional, tal como discriminación de las poblaciones migrantes ${ }^{(37)}$ y el dominio de la lengua de la región de destino ${ }^{(30)}$, podrían afectar esta relación. Aunque potencialmente relevante, la medición de discriminación en nuestro medio no es muy frecuente limitando la disponibilidad de instrumentos adecuados para tener una aproximación a este fenómeno social.

En el estudio de Mui et al. ${ }^{(30)}$ se evidencia que el dominio del idioma inglés en las personas de origen asiático es una determinante fundamental en la percepción de calidad de vida de los individuos migrantes en los Estados Unidos de Norteamérica. Yoon et al. ${ }^{(31)}$, utilizando también el dominio del lenguaje como una variable vinculada con aculturación, demuestra una relación directa entre la aculturación y el bienestar de salud. El dominio de la lengua castellana en el grupo de migrantes, evaluado como un proxy de aculturación, ha sido descrito en nuestro grupo como asociado positivamente con obesidad ${ }^{(38)}$. Sin embargo, dicha asociación es negativa al evaluar el lenguaje preferido para escuchar como marcador de aculturación, es decir, las oportunidades de ser obeso son menores si el migrante prefiere escuchar en Castellano ${ }^{(38)}$. Esto indica que la aculturación por sí misma es un proceso complejo. El análisis de aculturación solo puede ser evaluado en comparaciones únicamente entre migrantes, dado que son los migrantes los únicos que evidencian el cambio de adaptación que intenta medir la aculturación, lo que limita su aplicación en este estudio comparativo cuyo objetivo involucra una exploración conjunta con población no migrante.

Por otro lado, se ha reportado que la población latina migrante a la zona de Los Ángeles en Estados Unidos de Norteamérica tiene una exposición alta a violencia política, afectando su salud mental y calidad de vida ${ }^{(5)}$. Menos del $8 \%$ de este grupo de Latinos procedía del conglomeradodeindividuos calificadoscomoprocedentes de Honduras, Nicaragua, Cuba, y Sudamérica lo cual limita la interpretación de este estudio en nuestro medio. El estudio del PERU MIGRANT fue diseñado teniendo en cuenta la violencia política como un factor propulsor de migración ${ }^{(8)}$ y el contexto sociopolítico peruano.
Ciertas limitaciones en el presente estudio deberían reconocerse. Primero, resaltar que el presente estudio es un análisis secundario en una submuestra de un estudio cuyo diseño primario tuvo otros fines. A pesar de ello, se trata de un diseño que toma ventaja de los procesos de migración internos, sobre todo migración rural-urbana, que permite explorar distintos componentes vinculados al proceso de migración. Esto es de suma relevancia no solo para el Perú sino también para otros contextos similares de economías emergentes, en donde los procesos de urbanización vienen en aumento $(39,40)$, usualmente impulsados por procesos de migración interna.

Segundo, este análisis exploratorio no permite una evaluación en detalle del comportamiento de distintas variables en modelos multivariables. Las futuras hipótesis que puedan generarse deberían complementarse con el análisis de covariables discutidas en el presente trabajo y contemplar desde su diseño análisis estratificados según edad y sexo.

Tercero, la selección de los participantes no fue necesariamente aleatoria y se tomó ventaja de un proceso de reclutamiento mayor puesto en marcha para el estudio PERU MIGRANT ${ }^{(8)}$. Si bien esto podría introducir algunos potenciales sesgos, es decir, por ejemplo aquellos con mayores problemas de salud podrían haber tenido una mayor avidez por aceptar participar en el estudio, consideramos que estos serían mínimos y no-diferenciales. El estudio global (8) encontró perfiles similares a los reportados en este estudio con relación al nivel educativo alcanzado en población migrante y urbana, lo que nos indica que la submuestra analizada no es sustancialmente distinta y podría argumentarse que se trata de una muestra cuasialeatoria.

Cuarto, el diseño del presente estudio es de tipo transversal y, por ende, el fenómeno de causalidad reversa podría estar presente. Esto sugeriría que, contrario a lo postulado -en donde la migración es la que afecta a la calidad de vida-, es posible que la percepción negativa de calidad de vida sea lo que impulse la migración. Es decir, es válido plantear que el contexto de violencia sociopolítica del Perú en las últimas décadas haya afectado la calidad de vida de los pobladores, lo que a su vez haya tenido un impacto en las decisiones de migrar. Este último escenario lo consideramos improbable, sobre todo por la temporalidad de los eventos, dado que el estudio PERU MIGRANT tuvo como una de sus fortalezas el evaluar a migrantes que dejaron su lugar de origen a los 15 años de edad en promedio y que tenían en promedio 32 años residiendo en zona urbana ${ }^{\left({ }^{(8)}\right.}$. En tal sentido, los procesos migratorios son bastante establecidos y el 
reporte de calidad de vida captura las relaciones y percepciones del grupo migrante y urbano en relación con su entorno urbano en Lima.

Los resultados presentados en este estudio sirven como un primer alcance en la evaluación de la calidad de vida en población migrante procedente de una zona rural y establecida en una zona urbana en el Perú. Los hallazgos diferenciales según los dominios contemplados sugieren que la calidad de vida se comporta en un patrón más complejo de lo esperado. Los distintos dominios del instrumento utilizado capturan información diversa acerca de la propia persona, las relaciones interpersonales y el entorno del sujeto evaluado.

Si bien nuestros resultados no tienen la fuerza necesaria para dar conclusiones más contundentes, no deja de ser un primer alcance en un contexto que debería ser explorado con más detalle. En el futuro, exploraciones más detalladas podrían hacerse con el objetivo último de generar conocimientos que ayuden a mejorar la calidad de vida relacionada con la salud de las personas migrantes.

\section{AGRADECIMIENTOS}

Este trabajo fue financiado por Wellcome Trust (GR074833MA). CRONICAS - Centro de Excelencia en Enfermedades Crónicas de la UPCH es financiado por el National Heart, Lung and Blood Institute (NHLBI), National Institute of Health (NIH), Department of Health and Human Services, mediante contrato No. HHSN268200900033C. CLdM es trainee de CRONICAS. Nuestro agradecimiento en especial a nuestros colegas de la Universidad Peruana Cayetano Heredia y A.B. PRISMA en Lima, Perú y varios otros en el Reino Unido, así como al personal y equipo encargado del trabajo de campo que contribuyeron en diferentes etapas del estudio. Adicionalmente, nuestros sinceros agradecimientos a todas las personas que accedieron ser parte de este estudio así como a Juan Francisco Chiroque, Candice Romero y Lilia Cabrera que coordinaron la fase del trabajo de campo de este estudio.

\section{Fuentes de Financiamiento}

Este trabajo fue financiado por Wellcome Trust (GR074833MA). CRONICAS - Centro de Excelencia en Enfermedades Crónicas de la UPCH es financiado por el National Heart, Lung and Blood Institute (NHLBI), National Institute of Health (NIH), Department of Health and Human Services, mediante contrato No. HHSN268200900033C.

\section{Conflictos de Interés}

Los autores declaran no tener conflictos de interés en la publicación de este artículo.

\section{REFERENCIAS BIBLIOGRÁFICAS}

1. WHOQOL-Group. The World Health Organization Quality of Life assessment (WHOQOL): position paper from the World Health Organization. Soc Sci Med. 1995;41(10):1403-9.

2. Lopes AA, Bragg-Gresham JL, Satayathum S, McCullough K, Pifer T, Goodkin DA, et al. Health-related quality of life and associated outcomes among hemodialysis patients of different ethnicities in the United States: the Dialysis Outcomes and Practice Patterns Study (DOPPS). Am J Kidney Dis. 2003;41(3):605-15.

3. Mapes DL, Lopes AA, Satayathum S, McCullough KP, Goodkin DA, Locatelli F, et al. Health-related quality of life as a predictor of mortality and hospitalization: the Dialysis Outcomes and Practice Patterns Study (DOPPS). Kidney Int. 2003;64(1):339-49.

4. Sullivan PW, Nelson JB, Mulani PM, Sleep D. Quality of life as a potential predictor for morbidity and mortality in patients with metastatic hormone-refractory prostate cancer. Qual Life Res. 2006;15(8):1297-306.

5. Eisenman DP, Gelberg L, Liu H, Shapiro MF. Mental health and health-related quality of life among adult Latino primary care patients living in the United States with previous exposure to political violence. JAMA. 2003;290(5):627-34.

6. Pando C. ¿Quienes son los desplazados? Análisis de los resultados de la encuesta a desplazados por efectos de la violencia política (Censo por la Paz 2001). Lima: Ministerio de la Mujer y Desarrollo Social; 2003.

7. Instituto Nacional de Estadística e Informática. Migraciones internas en el Perú. Lima: INEl; 1995.

8. Miranda JJ, Gilman RH, Garcia HH, Smeeth L. The effect on cardiovascular risk factors of migration from rural to urban areas in Peru: PERU MIGRANT Study. BMC Cardiovasc Disord. 2009;9:23.

9. WHOQOL-Group. The World Health Organization Quality of Life Assessment (WHOQOL): development and general psychometric properties. Soc Sci Med. 1998;46(12):1569-85.

10. WHOQOL-Group. Development of the World Health Organization WHOQOL-BREF quality of life assessment. The WHOQOL Group. Psychol Med. 1998;28(3):551-8.

11. World Health Organization. WHOQOL-BRIEF: Introduction, administration, scoring and generic version of the assessment. Geneva: WHO; 1996.

12. Crosby RD, Kolotkin RL, Williams GR. Defining clinically meaningful change in health-related quality of life. J Clin Epidemiol. 2003;56(5):395-407.

13. Hays RD, Woolley JM. The concept of clinically meaningful difference in health-related quality-of-life research. How meaningful is it? Pharmacoeconomics. 2000;18(5):419-23.

14. Hays RD, Farivar SS, Liu H. Approaches and recommendations for estimating minimally important differences for health-related quality of life measures. COPD. 2005;2(1):63-7. 
15. Schunemann HJ, Guyatt GH. Commentary--goodbye M(C)ID! Hello MID, where do you come from? Health Serv Res. 2005;40(2):593-7.

16. Cohen J. Statistical power analysis in the behavioral sciences. $2^{\text {nd }}$ ed. Hillsdale, NJ: Lawrence Erlbaum Associates, Inc; 1988.

17. Masterson Creber RM, Smeeth L, Gilman RH, Miranda JJ. Physical activity and cardiovascular risk factors among rural and urban groups and rural-to-urban migrants in Peru: a cross-sectional study. Rev Panam Salud Publica. 2010;28(1):1-8.

18. Galea S, Freudenberg N, Vlahov D. Cities and population health. Soc Sci Med. 2005;60(5):1017-33.

19. United Nations, Department of Economic and Social Affairs, Population Division. International Migration Report. New York: United Nations; 2002.

20. Chackiel J. La dinámica demográfica en América Latina. Serie Población y Desarrollo $N^{\circ}$ 52. Santiago de Chile: CELADE/CEPAL; 2004

21. Razum O. Commentary: Of salmon and time travellersmusing on the mystery of migrant mortality. Int J Epidemiol. 2006;35(4):919-21.

22. Razum O, Twardella D. Time travel with Oliver Twist--towards an explanation foa a paradoxically low mortality among recent immigrants. Trop Med Int Health. 2002;7(1):4-10.

23. Abraido-Lanza AF, Dohrenwend BP, Ng-Mak DS, Turner JB. The Latino mortality paradox: a test of the "salmon bias" and healthy migrant hypotheses. Am J Public Health. 1999;89(10):1543-8

24. McKay L, Macintyre S, Ellaway A. Migration and health: a review of the international literature. Occasional paper No. 12. Glasgow: Medical Research Council, Social \& Public Health Sciences Unit, University of Glasgow; 2003.

25. Jacoby E, Bull F, Neiman A. Cambios acelerados de estilo de vida obligan a fomentar la actividad física como prioridad en la Región de las Américas. Rev Panam Salud Publica. 2003;14(4):223-5.

26. CEPAL. América Latina: proyecciones de población urbana y rural 1970 - 2025. Boletín Demográfico. 2005;38(76): 1-255.

27. United Nations Population Fund. UNFPA state of world population 2007: unleashing the potential of urban growth. New York: UNFPA; 2007.

28. Comisión de la Verdad y Reconciliación. Informe Final de la Comisión de la Verdad y Reconciliación. Lima: Comisión de la Verdad y Reconciliación; 2003.
29. McGuire J, Li X, Wang B. Social stigma and quality of life among rural-to-urban migrants in China: a comparison with their rural counterparts. World Health Popul. 2009;11(2):30-41.

30. Mui AC, Kang SY, Kang D, Domanski MD. English language proficiency and health-related quality of life among Chinese and Korean immigrant elders. Health Soc Work. 2007;32(2):119-27.

31. Yoon E, Lee RM, Goh M. Acculturation, social connectedness, and subjective well-being. Cultur Divers Ethnic Minor Psychol. 2008;14(3):246-55.

32. Muennig P, Fahs MC. Health status and hospital utilization of recent immigrants to New York City. Prev Med. 2002;35(3):225-31.

33. McDonald JT, Kennedy S. Insights into the 'healthy immigrant effect': health status and health service use of immigrants to Canada. Soc Sci Med. 2004;59(8):1613-27.

34. Newbold KB, Danforth J. Health status and Canada's immigrant population. Soc Sci Med. 2003;57(10):1981-95.

35. Sundquist J. Migration, equality and access to health care services. J Epidemiol Community Health. 2001;55(10):691-2.

36. Farley T, Galves A, Dickinson LM, Perez Mde J. Stress, coping, and health: a comparison of Mexican immigrants, Mexican-Americans, and non-Hispanic whites. J Immigr Health. 2005;7(3):213-20.

37. Zhang J, Li X, Fang X, Xiong Q. Discrimination experience and quality of life among rural-to-urban migrants in China: the mediation effect of expectation-reality discrepancy. Qual Life Res. 2009;18(3):291-300.

38. Bernabe-Ortiz A, Gilman RH, Smeeth L, Miranda JJ. Migration surrogates and their association with obesity among within-country migrants. Obesity (Silver Spring). 2010;18(11):2199-203.

39. Van Ginkel H. Urban future. Nature. 2008;456(n1s):32-3.

40. Daar AS, Singer PA, Leah Persad D, Pramming SK, Matthews DR, Beaglehole R, et al. Grand challenges in chronic non-communicable diseases. Nature. 2007;450(7169):494-6

Correspondencia: J. Jaime Miranda, MD, MSc, PhD.

Dirección: Av. Armendáriz 497, 2do Piso, Miraflores, Lima 18, Perú.

Teléfono: (511) 241-6978.

Correo electrónico: Jaime.Miranda@upch.pe

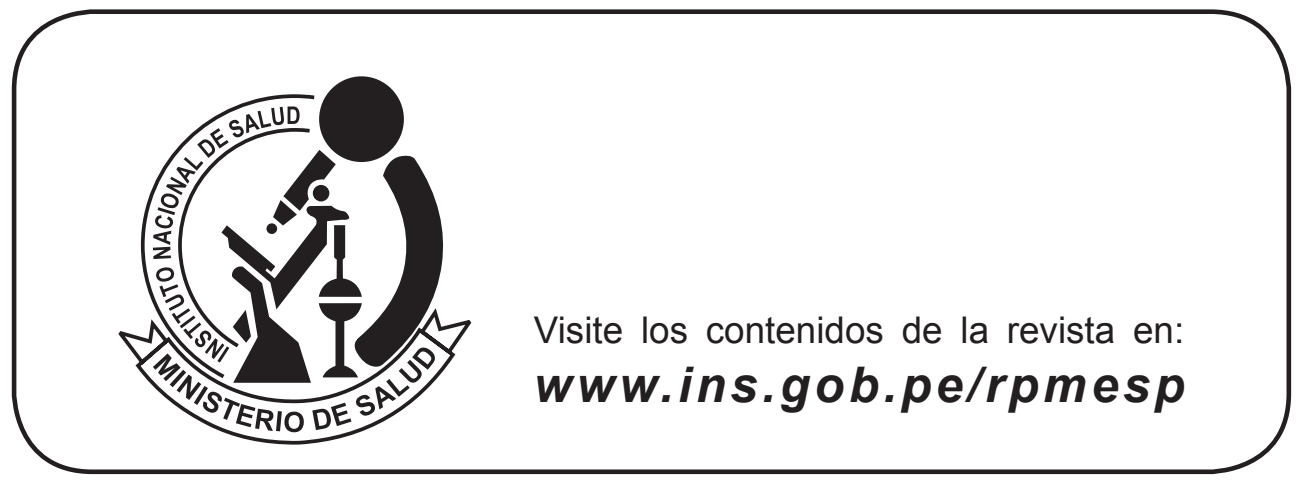

